\title{
ATRIAL FIBRILLATION
}

\section{Physician specialty and ICD implant outcome}

Curtis et al. reveal that, compared with implantable cardioverter-defibrillator (ICD) implantation performed by electrophysiologists, procedures carried out by nonelectrophysiologists are prone to more complications.

Nonelectrophysiologist physicians are trained to implant ICDs to prevent delays (when electrophysiologists are unavailable) for patients who require this potentially lifesaving treatment. The Heart Rhythm Society suggests that training be standardized, as differences in training, experience, and technique could all affect post-treatment outcomes. In addition, some physicians may be unfamiliar with clinical indications for patients who could benefit from a defibrillator with cardiac resynchronization therapy (CRT-D).

Curtis and colleagues analyzed data from the registry of all ICD implantation procedures for primary prevention of sudden cardiac death in hospitals in the
US. The majority of procedures were carried out by electrophysiologists who had an overall complication rate of $3.5 \%$. Nonelectrophysiologist cardiologists (21.9\% of implantations), thoracic surgeons (1.7\%), and other specialists (5.5\%) had overall complication rates of $4.0 \%, 5.8 \%$ and $4.0 \%$, respectively. Electrophysiologists were more likely to implant a CRT-D in patients who met criteria compared with the other physicians.

The researchers concluded that confirmation of these findings could warrant a review of training recommendations for those who are nonelectrophysiologists.

Lisa Richards

Original article Curtis, J. P. et al. Association of physician certification and outcomes among patients receiving an implantable cardioverter-defibrillator. JAMA 301, 1661-1670 (2009). 